\title{
Magnetic Properties of Liquid RE-Co (RE = Dy, Er) Alloys
}

\author{
Satoru Ohno ${ }^{1, *}$, Hironori Shimakura ${ }^{1}$, Shuta Tahara $^{2}$, and Tatsuya Okada $^{3}$ \\ ${ }^{1}$ Niigata University of Pharmacy and Applied Life Sciences, Niigata 956-8603, Japan \\ ${ }^{2}$ Department of Physics and Earth Sciences, Faculty of Science, University of the Ryukyus, Okinawa \\ 903-0213, Japan \\ ${ }^{3}$ Niigata College of Technology, Niigata 950-2076, Japan
}

\begin{abstract}
The magnetic susceptibilities $\chi$ of liquid Dy-Co and Er-Co alloys have a large and negative temperature coefficient, which suggests that the Dy, Er, and Co ions in their liquid alloys are in the magnetic state. However, the temperature dependence of $\chi$ in both systems becomes weak near the content of $70 \mathrm{at} \% \mathrm{Co}$. It is interesting that the compositional dependence of $\chi$ for liquid Dy-Co and Er-Co alloys has a minimum at content of 80 at $\%$ Co, respectively. On the Co-rich side, the magnetic susceptibility of liquid Dy-Co and Er-Co alloys obeyed the Curie-Weiss law with regard to their temperature dependence of $\chi$. On the rare earth-rich side, the magnetic susceptibilities of liquid Dy-Co and Er-Co alloys also exhibited Curie behavior with a reasonable value for the effective number of Bohr magnetons. The compositional dependence of $\chi_{4 \mathrm{f}}$ for liquid Dy-Co and ErCo alloys was extracted by subtracting the corresponding data for liquid La-Co alloys from $\chi$ for the liquid Dy-Co and Er-Co alloys, respectively.
\end{abstract}

\section{Introduction}

Busch and colleagues [1] reported the electronic properties of liquid transition metal (TM), rare earth metal (RE), and their alloys. Schlapbach [2] reported more detailed dependence of magnetic susceptibility on composition with a minimum for liquid Ce-TM (TM $=\mathrm{Fe}, \mathrm{Co}$, $\mathrm{Ni}$ ) alloys and found a continuous transition of $\mathrm{Ce}$ ions into the nonmagnetic state. Moreover, Singer et al. [3] extended and explained the compositional dependence of $\chi$ for liquid $\mathrm{Ce}-\mathrm{TM}, \mathrm{Pr}-\mathrm{TM}$, La-TM (TM = Fe, Co, Ni), and Sm-Fe alloys. In their paper [3], the compositional dependence of $\chi$ for liquid La-TM alloys plays an important role in understanding the magnetic susceptibility of liquid RE-TM $(\mathrm{RE}=\mathrm{Ce}, \mathrm{Pr}, \mathrm{Sm})$ alloys. Actually, the compositional dependence of $\chi_{4 \mathrm{f}}$ can be obtained by subtracting the corresponding data for liquid La-TM alloys from $\chi$ for liquid RE-TM $(\mathrm{RE}=\mathrm{Ce}, \mathrm{Pr}, \mathrm{Sm})$ alloys.

We attempt to obtain the magnetic susceptibilities as the functions of temperature and composition for liquid Dy-Co and Er-Co alloys. The magnetic susceptibility of liquid DyCo and Er-Co alloys consists of the two large terms due to the $3 \mathrm{~d}$ - and $4 \mathrm{f}$-electron states.

*Corresponding author: ohno@nupals.ac.jp 
The contribution of these two electron states provides a convenient sectionalization in the each region on the Co-rich and RE-rich $(\mathrm{RE}=\mathrm{Dy}, \mathrm{Er})$ sides. Therefore, these two terms can be separated to obtain the temperature dependence of $\chi_{3 \mathrm{~d}}$ and $\chi_{4 \mathrm{f}}$ for liquid RE-Co $(\mathrm{RE}=$ Dy, Er) alloys. The $P_{\text {eff }}$ values for the Dy, Er, and Co ions in liquid Dy-Co and Er-Co alloys can thus be estimated from the temperature dependence of $\chi_{3 \mathrm{~d}}$ and $\chi_{4 \mathrm{f}}$. We investigate the trend of $P_{\text {eff }}$ for Dy and Er ions on the basis of the s-f and indirect d-f interactions and compare the trend of Peff for Ce, Pr, Gd, Dy, and Er ions in liquid RE-Co alloys (RE $=\mathrm{Ce}$, Pr, Gd, Dy, Er), respectively.

\section{Experimental}

Magnetic susceptibility measurements were made using the Faraday method with a torsion balance $[4,5]$. The force applied to the sample in the cell was quantified by an automatic method of feedback current control. Mohr's salt $\left(\chi=1.26 \times 10^{-2} \mathrm{~cm}^{3} / \mathrm{mol}\right.$ at room temperature) was employed as a standard sample. The field strength $H$ of the electromagnet was $10 \mathrm{kOe}$ with a $6.0 \mathrm{~cm}$ gap between pole pieces, and $H \cdot \mathrm{d} H / \mathrm{d} x$ was $6.25 \pm 0.12$ $(\mathrm{kOe})^{2} / \mathrm{cm}$.

The measurement system can be evacuated in a vacuum of $1.2 \times 10^{-2} \mathrm{~Pa}$ and filled with a purified $\mathrm{Ar}$ gas at a pressure of $7 \mathrm{kPa}$. The RE-Co alloy sample was placed in a magnesia $(\mathrm{MgO})$ crucible packed loosely with a $\mathrm{MgO}$ rod, and was completely melted and homogenized in a silicon carbide furnace under the above condition. The measurements of liquid $\mathrm{Co}$ and Er were carried out up to about $1570{ }^{\circ} \mathrm{C}$. The measurements on the Co-rich side were carried out from the temperature between $1500{ }^{\circ} \mathrm{C}$ and $1550{ }^{\circ} \mathrm{C}$ to the melting point. The chemical composition of each sample was determined by accurately measuring the mass of each component before mixing. After this measurement, the decrease in weight of a $0.2 \mathrm{~g}$ sample was less than $4 \mathrm{mg}$. The temperature of the liquid alloy was determined using Pt-Pt13\%Rh thermocouples placed close to the sample. The elemental purity was $99.9 \%$ for Dy, Er, and Co.

\section{Results}

As shown in Fig. 1(a), liquid Co has a large and negative temperature coefficient of magnetic susceptibility. On the Co-rich side, the magnetic susceptibilities $\chi$ of liquid DyCo alloys decrease with increasing Dy content up to 20 at $\%$ Dy and these temperature coefficients of $\chi$ become weak up to 30 at $\%$ Dy. With further increasing Dy content, the magnetic susceptibilities of liquid Dy-Co alloys increase with a large and negative temperature coefficient of $\chi$. This suggests that the Co and Dy ions in the liquid Dy-Co alloys are in magnetic states. The values of $\chi$ for liquid Co and Dy are in good agreement with those obtained in the previous works $[5,6]$.

As shown in Fig. 1(b), the magnetic susceptibilities $\chi$ of liquid Er-Co alloys also decrease with increasing Er content up to 20 at $\%$ Er and these temperature coefficients of $\chi$ become weak up to 30 at $\%$ Er. With further increasing Er content, similarly, the magnetic susceptibilities of liquid Er-Co alloys increase with a large and negative temperature coefficient of $\chi$. This suggests that the Co and Er ions in the liquid Er-Co alloys are in magnetic states. The limit of utility of $\mathrm{MgO}$ crucible may be up to the temperature of 1570 ${ }^{\circ} \mathrm{C}$ for liquid Er.

Figure 2 shows the compositional dependence of $\chi$ for liquid Dy-Co and Er-Co alloys at 1400 and $1500{ }^{\circ} \mathrm{C}$. We added the compositional dependence of $\chi$ of liquid Gd-Co and La-Co alloys at 1350 and $1500{ }^{\circ} \mathrm{C}$ to do some more investigating. The compositional 
dependences of $\chi$ for liquid Dy-Co and Er-Co alloys decrease with decreasing Co content and exhibit a minimum at the compositions of 20 at $\%$ Dy and Er, respectively. The magnetic susceptibilities of liquid Dy, Er, and Gd are approximately 901, 693, and $663 \times$ $10^{-5} \mathrm{~cm}^{3} / \mathrm{mol}$ at the melting point, respectively.
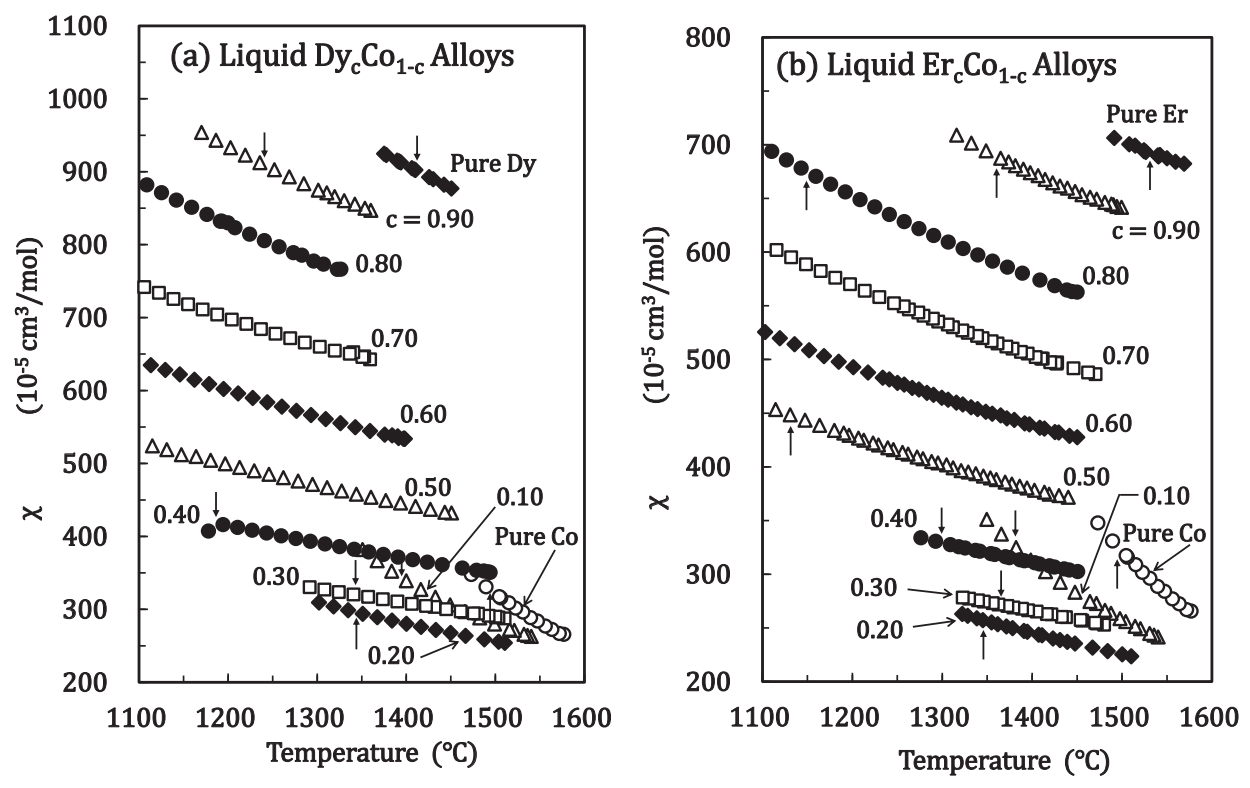

Fig. 1. Magnetic susceptibility of liquid $\mathrm{RE}_{c} \mathrm{Co}_{1-c}$ alloys as a function of temperature. (a) liquid $\mathrm{Dy}_{c} \mathrm{Co}_{1-c}$ alloys; (b) liquid $\mathrm{Er}_{c} \mathrm{Co}_{1-c}$ alloys. The arrows indicate the melting point of the alloys. Experimental error is about $2.5 \%$.

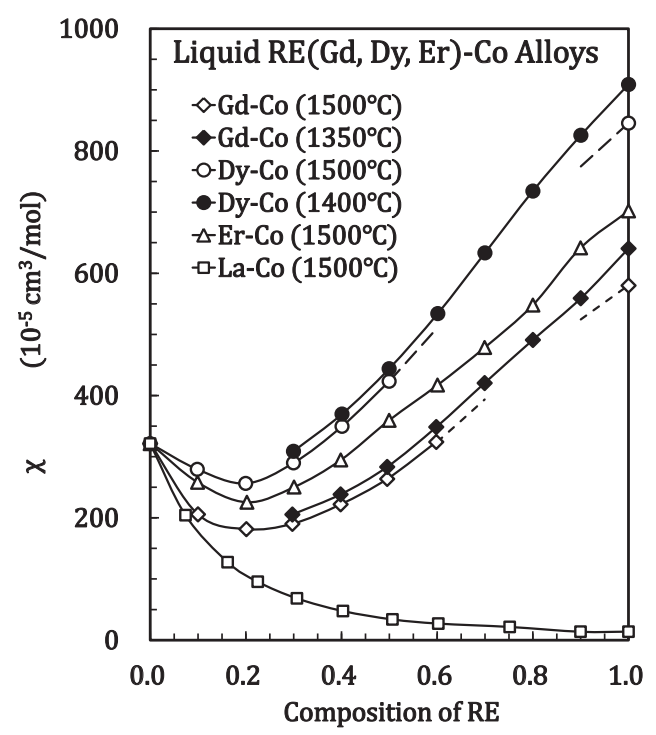

Fig. 2. Dependence of $\chi$ on composition for $\mathrm{RE}_{c} \mathrm{Co}_{1-c}(\mathrm{ER}=\mathrm{Dy}, \mathrm{Er}, \mathrm{Gd})$ alloys. The compositional dependence of $\chi$ for liquid $\mathrm{La}_{c} \mathrm{Co}_{1-c}$ alloys was reported by Singer et al. [3]. The solid lines and dashed lines are drawn as guides for the eye. 


\section{Discussion}

The magnetic susceptibility of a liquid RE-Co alloy is given by $[5,7]$

$$
\chi_{1}=\left[(1-c) \chi_{\mathrm{dia}}\left(\mathrm{Co}^{+}\right)+c \chi_{\mathrm{dia}}\left(\mathrm{RE}^{3+}\right)\right]+\chi_{\mathrm{el}}+\chi_{3 \mathrm{~d}}+\chi_{4 \mathrm{f}}+\chi_{5 \mathrm{~d}},
$$

where $\chi_{\text {dia }}\left(\mathrm{Co}^{+}\right)$and $\chi_{\text {dia }}\left(\mathrm{RE}^{3+}\right)$ are the diamagnetic susceptibilities due to the $\mathrm{Co}^{+}$and $\mathrm{RE}^{3+}$ $(\mathrm{RE}=\mathrm{Dy}, \mathrm{Er}, \mathrm{Gd})$ ions, respectively. The diamagnetic susceptibilities of $\mathrm{Co}^{+}, \mathrm{Dy}^{3+}, \mathrm{Er}^{3+}$, and $\mathrm{Gd}^{3+}$ ions have been estimated by Bain and Berry [8], and Mendelsohn et al. [9], respectively. The diamagnetic susceptibility of Co ions in metals has been obtained by Banhart et al. [10]. The values of $\chi_{\text {dia }}$ for $\mathrm{Co}^{+}, \mathrm{Dy}^{3+}, \mathrm{Er}^{3+}$, and $\mathrm{Gd}^{3+}$ ions employed in this work are $-1.82,-1.90,-1.80$, and $-1.80 \times 10^{-5} \mathrm{~cm}^{3} / \mathrm{mol}$, respectively. We assume that the total $\chi_{\text {dia }}$ for liquid Dy-Co, Er-Co, and Gd-Co alloys varies linearly with composition.

$\chi_{\mathrm{el}}$ is the paramagnetic susceptibility due to conduction electrons, expressed as $[7,11]$

$$
\chi_{\mathrm{el}}=\alpha\left(m^{*}, r_{\mathrm{s}}\right) \mu_{\mathrm{B}}^{2} N\left(E_{\mathrm{F}}\right),
$$

where $N\left(E_{\mathrm{F}}\right)$ is the density of states at $E_{\mathrm{F}}$, which can be estimated from the nearly free electron model as follows: $[7,11]$

$$
N\left(E_{\mathrm{F}}\right)=\left(V_{\mathrm{a}} / 2 \pi^{2}\right)\left(2 m / \hbar^{2}\right)^{3 / 2}{E_{\mathrm{F}}}^{1 / 2} .
$$

Here, $V_{\mathrm{a}}$ is the atomic volume and $E_{\mathrm{F}}$ is given by $[7,11]$

$$
E_{\mathrm{F}}=\left(\hbar^{2} / 2 m\right)\left(3 \pi^{2} N_{\mathrm{e}} / V_{\mathrm{a}}\right)^{2 / 3},
$$

where $N_{\mathrm{e}}$ is the number of conduction electrons.

We assume that the $E_{\mathrm{F}}$ and $N\left(E_{\mathrm{F}}\right)$ values are estimated by the linear interpolation of $N_{\mathrm{e}}$ and $V_{\mathrm{a}}$ from the RE side to the Co side. The $4 \mathrm{~s}$ electrons of Co ions and the $6 \mathrm{~s}^{2}$ electrons of $\mathrm{RE}$ ions are simply treated as conduction electrons. It is assumed that the $5 \mathrm{~d}$ electrons of $\mathrm{RE}$ ions form independently the density of $5 \mathrm{~d}$-electron states.

We assume that the $\mathrm{m}^{*} / \mathrm{m}$ ratio is 1.0 in liquid Co, RE, and their alloys. In this assumption, the enhancement factor for nearly free electrons is given by $[12,13]$

$$
\alpha\left(m, r_{\mathrm{s}}\right)=\alpha\left(r_{\mathrm{s}}\right)-1 / 3
$$

where $\alpha\left(r_{\mathrm{s}}\right)$ is the enhancement factor of free-electron theory. The dimensionless quantity $r_{\mathrm{s}}$ is defined as $\left(3.64 \times 10^{8}\right) / k_{\mathrm{F}}$, where $k_{\mathrm{F}}$ is the Fermi wave number. The $\alpha\left(r_{\mathrm{s}}\right)$ estimated by the random phase approximation increases slightly with increase $r_{\mathrm{s}}$, and were determined to be $1.28,1.28,1.28$, and 1.27 for liquid Dy, Er, Gd, and Co, respectively.

The compositional dependences of $\chi_{\mathrm{el}}$ estimated using this approach deviate above slightly from the straight line connected on the Co side and $\mathrm{RE}(\mathrm{RE}=\mathrm{Dy}, \mathrm{Er}, \mathrm{Gd})$ side, respectively. Assuming that the value of $\chi_{\text {dia }}$ for liquid RE-Co varies linearly with composition, we estimated the compositional dependence of $\left(\chi_{\text {dia }}+\chi_{\mathrm{el}}\right)$, as shown in Fig. 3 . As a result, the compositional dependence of $\left(\chi_{\text {dia }}+\chi_{\mathrm{el}}\right)$ obtained in this procedure approximates a straight line on the scale shown in Fig. 3. As a result, the compositional dependence of $\left(\chi_{\text {dia }}+\chi_{\mathrm{el}}\right)$ obtained in this procedure approximates a straight line on the scale shown in Fig. 3. The values of $V_{\mathrm{a}}, \chi_{\mathrm{dia}}$, and $\chi_{\mathrm{el}}$ are shown in Table 1. 

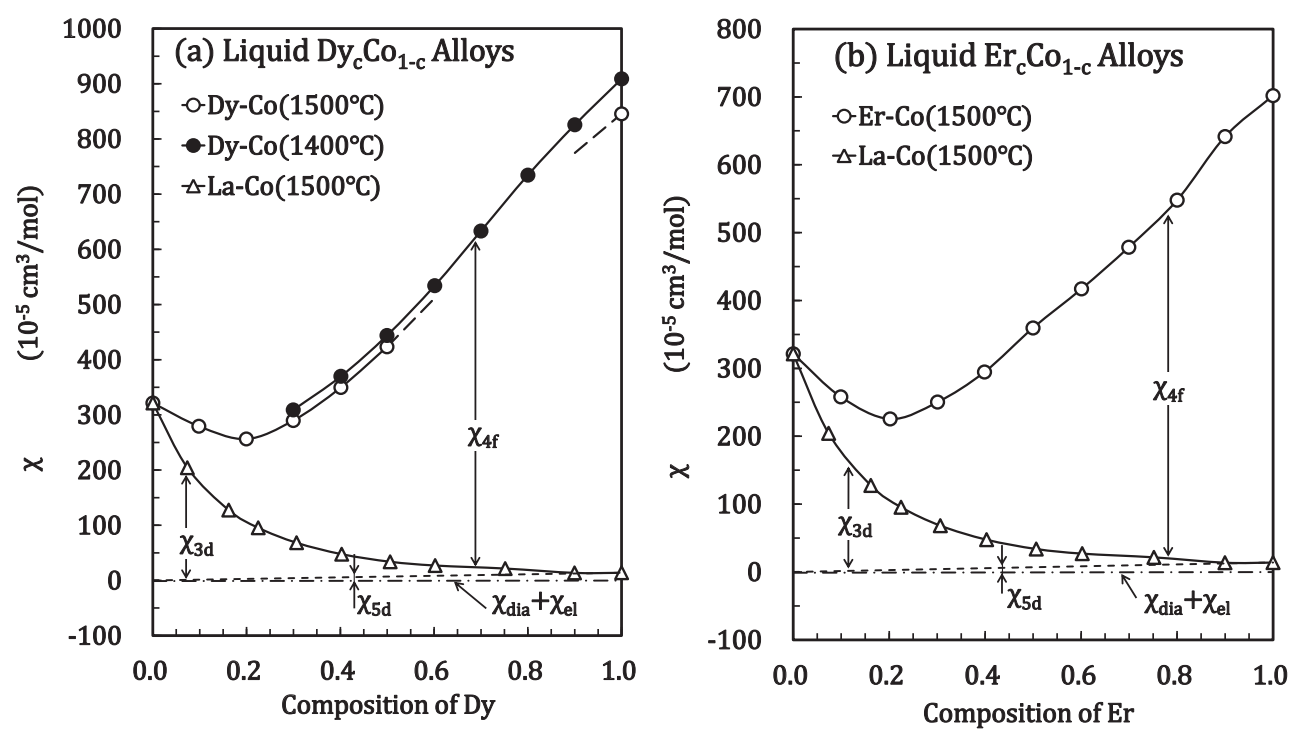

Fig. 3. Dependence of $\chi$ on composition for liquid $\mathrm{RE}_{c} \mathrm{Co}_{1-c}$ alloys. (a) liquid $\mathrm{Dy}_{c} \mathrm{Co}_{1-c}$ alloys; (b) liquid $\mathrm{Er}_{c} \mathrm{Co}_{1-c}$ alloys. The compositional dependence of $\chi$ for liquid $\mathrm{La}_{c} \mathrm{Co}_{1-c}$ alloys was reported by Singer et al. [3]. The base line is the compositional dependence of $\left(\chi_{\mathrm{dia}}+\chi_{\mathrm{el}}\right)$. The solid lines and dashed lines are drawn as guides for eye.

Table 1. $V_{\text {a }}, \chi_{\text {dia }}$, and $\chi_{\text {el }}$ for $\mathrm{Co}^{+}, \mathrm{Dy}^{3+}, \mathrm{Er}^{3+}, \mathrm{La}^{3+}, \mathrm{Gd}^{3+}, \mathrm{Pr}^{3+}$, and $\mathrm{Ce}^{3+}$ ions.

\begin{tabular}{|c|c|c|c|c|c|c|c|}
\hline & $\mathrm{Co}^{+}$ & $\mathrm{Dy}^{3+}$ & $\mathrm{Er}^{3+}$ & $\mathrm{La}^{3+}$ & $\mathrm{Gd}^{3+}$ & $\mathrm{Pr}^{3+}$ & $\mathrm{Ce}^{3+}$ \\
\hline$V_{\mathrm{a}}\left(10^{-23} \mathrm{~cm}^{3} / \mathrm{mol}\right)$ & 1.26 & 3.32 & 3.32 & 3.94 & 3.80 & 3.63 & 3.56 \\
\hline$\chi_{\mathrm{EXP}}\left(10^{-5} \mathrm{~cm}^{3} / \mathrm{mol}\right)$ & 321 & 846 & 702 & 14 & 579 & 147 & 85 \\
\hline$\chi_{\text {dia }}\left(10^{-5} \mathrm{~cm}^{3} / \mathrm{mol}\right)$ & -1.82 & -1.90 & -1.80 & -2.00 & -2.00 & -2.00 & -2.00 \\
\hline$\chi_{\mathrm{el}}\left(10^{-5} \mathrm{~cm}^{3} / \mathrm{mol}\right)$ & 0.68 & 1.65 & 1.65 & 1.86 & 1.81 & 1.75 & 1.87 \\
\hline$\chi_{\text {dia }}+\chi_{\mathrm{el}}\left(10^{-5} \mathrm{~cm}^{3} / \mathrm{mol}\right)$ & -1.14 & -0.25 & -0.15 & -0.14 & -0.19 & -0.25 & -0.13 \\
\hline
\end{tabular}

As mentioned above, the 5d-electrons of RE and La ions seems to form independently the density of $5 \mathrm{~d}$-electron states. Therefore, the magnetic susceptibility of $\chi_{5 \mathrm{~d}}$ due to $5 \mathrm{~d}-$ electron is given by [14]

$$
\chi_{5 \mathrm{~d}}=N_{\mathrm{A}} \mu_{\mathrm{B}}^{2} \rho_{5 \mathrm{~d}}\left(E_{\mathrm{F}}\right) /\left[1-U \rho_{5 \mathrm{~d}}\left(E_{\mathrm{F}}\right) / 2\right],
$$

where $U$ is the intra-atomic Coulomb interaction. The experimental $\chi$ value of liquid La corresponds to $\left(\chi_{\mathrm{dia}}+\chi_{\mathrm{el}}+\chi_{5 \mathrm{~d}}\right)$ value. We can obtain the $\rho_{5 \mathrm{~d}}\left(E_{\mathrm{F}}\right)$ value of liquid La under the assumption that $U=2.0 \mathrm{eV}$. The $\rho_{5 \mathrm{~d}}\left(E_{\mathrm{F}}\right)$ obtained is $0.81 \mathrm{eV}^{-1} \cdot$ atom ${ }^{-1}$, which is larger than the $\rho_{6 \mathrm{~s}}\left(E_{\mathrm{F}}\right)$ value of $0.60 \mathrm{eV}^{-1} \cdot$ atom $^{-1}$. The $\rho_{5 \mathrm{~d}}\left(E_{\mathrm{F}}\right)$ value may be really admixed by $\rho_{6 \mathrm{~s}}\left(E_{\mathrm{F}}\right)$, which forms the width of $5 \mathrm{~d}$-state. The magnetic susceptibilities of liquid TM-La alloys consists of $\left(\chi_{\mathrm{dia}}+\chi_{\mathrm{el}}\right)$ value and $\chi_{5 \mathrm{~d}}$ of eq. (7). The dependence of $\left(\chi_{\mathrm{dia}}+\chi_{\mathrm{el}}+\chi_{5 \mathrm{~d}}\right)$ on composition obtained in this procedure approximates a straight line on the scale shown in Fig. 3. 
According to their suggestion of March and Sayers [15], the magnetic susceptibility of liquid Dy-Co, Er-Co, and Gd-Co alloys consists of the two large terms due to the $3 \mathrm{~d}$ electron and $4 \mathrm{f}$-electron states on the TM-rich side and RE-rich side. We compare the magnetic susceptibility of liquid RE-Co and La-Co alloys. The magnetic susceptibility of La-Co alloy is given by $[5,7]$

$$
\chi_{2}=\left[(1-c) \chi_{\mathrm{dia}}\left(\mathrm{Co}^{+}\right)+c \chi_{\mathrm{dia}}\left(\mathrm{La}^{3+}\right)\right]+\chi_{\mathrm{el}}+\chi_{3 \mathrm{~d}}+\chi_{5 \mathrm{~d}},
$$

where $\chi_{\text {dia }}\left(\mathrm{La}^{3+}\right)$ are the diamagnetic susceptibilities due to $\mathrm{La}^{3+}$ ions. Roughly speaking, we can obtain the $\chi_{4 \mathrm{f}}$ value by subtracting eq. (7) from eq. (1) as follows;

$$
\chi_{4 \mathrm{f}} \approx \chi_{1}-\chi_{2} \text {. }
$$

Assuming that $\left(\chi_{\mathrm{dia}}+\chi_{\mathrm{el}}\right)$ is considerably smaller than $\chi_{3 \mathrm{~d}}$ and $\chi_{4 \mathrm{f}}$, we can obtain the $\chi_{4 \mathrm{f}}$ values of eq. (8) with a high degree of accuracy.

Figure 3(a) shows the dependence of $\chi$ on composition for liquid Dy-Co alloys. Assuming that the $\chi_{3 \mathrm{~d}}$ of Co ions in liquid Dy-Co alloys may be little different from that in liquid La-Co alloys, we also plotted the dependence of $\chi$ on composition for liquid La-Co reported by Singer et al. [3]. This difference between the lines of liquid Dy-Co and La-Co alloys corresponds to the dependence of $\chi_{4 \mathrm{f}}$ on composition due to $4 \mathrm{f}$-electrons. The base line exhibits the dependence of $\left(\chi_{\text {dia }}+\chi_{\mathrm{el}}\right)$ on composition due to the Dy and Co ions, and conduction electrons, which is a very small value compared with $\chi_{4 \mathrm{f}}$ and $\chi_{3 \mathrm{~d}}$. The dependence of $\chi_{3 \mathrm{~d}}$ on composition decreases rapidly with decreasing Co content. The variation of $\chi_{4 \mathrm{f}}, \chi_{5 \mathrm{~d}}$, and $\chi_{3 \mathrm{~d}}$ are reasonable for the composition of Dy and Co.

Figure $3(\mathrm{~b})$ shows the dependence of $\chi$ on composition for liquid Er-Co alloys. The dependence of $\chi$ on composition for liquid La-Co alloys exhibits the data reported by Singer et al. [3]. As shown in Fig. 3(b), the significant point of comparison is that the difference between the lines for liquid Er-Co and La-Co increases with increasing Er (or La) content. This difference corresponds to the dependence of $\chi_{4 \mathrm{f}}$ on composition due to $4 \mathrm{f}$ electrons. The base line exhibits the dependence of $\left(\chi_{\text {dia }}+\chi_{\mathrm{el}}\right)$ on composition due to the $\mathrm{Er}$ (or $\mathrm{La}$ ) and Co ions, and conduction electrons, which is a very small value compared with $\chi_{4 \mathrm{f}}$ and $\chi_{3 \mathrm{~d}}$. The dependence of $\chi_{3 \mathrm{~d}}$ on composition decreases rapidly with decreasing Co content. The variations of $\chi_{4 \mathrm{f}}$ and $\chi_{3 \mathrm{~d}}$ obtained in Fig. 3(b) are also reasonable for the composition of Er and Co.

Figure 4 shows the dependence of $\chi_{4 \mathrm{f}}$ on composition for liquid RE-Co (RE $=\mathrm{Dy}$, Er, $\mathrm{Gd})$ alloys. For liquid Gd-Co alloys, the dependence of $\chi_{4 \mathrm{f}}$ on composition was obtained by similar manner. Liquid RE-Co alloys have similar dependence of $\chi_{4 \mathrm{f}}$ on composition, which deviates below from straight line. The $\chi_{4 \mathrm{f}}$ values of liquid RE-Co alloys decrease with increasing temperature, which reflects the temperature dependence of the experimental $\chi$ value. It suggests that the RE ions in liquid RE-Co alloys are in the magnetic state over the wide composition range. It is known that the Curie-Weiss law describes the magnetic susceptibility in the magnetic state fairly well. For liquid RE-Co in the magnetic state, the third and fourth terms on the right-hand side of eq. (1) is given by [5, 7]

$$
\chi_{4 \mathrm{f}, \text { or } 3 \mathrm{~d}}=\left[N_{\mathrm{A}} P_{\text {eff }}^{2} \mu_{\mathrm{B}}^{2} / 3 k_{\mathrm{B}}(T-\theta)\right]+\alpha,
$$

where $N_{\mathrm{A}}$ is the number of RE or Co atoms per mole. $P_{\text {eff }}$ is the effective number of Bohr magnetons. The symbols $\theta$ and $\alpha$ are the paramagnetic Curie temperature and a residual susceptibility at infinite temperature, respectively [9].

In this work, the $P_{\text {eff }}$ values of RE ions in liquid RE-Co alloys were obtained from the equation of $P_{\text {eff }}\left(\mathrm{RE}^{3+}\right)=\left(3 k_{\mathrm{B}} T \chi_{4 \mathrm{f}} / N_{\mathrm{A}}\right)^{1 / 2} \mu_{\mathrm{B}}$ under the assumptions that $\theta=0$ and $\alpha=0$. On 
the Co side, the $P_{\text {eff }}$ and $\theta$ for the Co ions in liquid RE-Co (RE $=$ Dy, Er, Gd) alloys were obtained from the plots of $\left(1 / \chi_{3 \mathrm{~d}}\right)$ vs $T$. The $\theta$ values obtained from this approach are 1450 , 1340 , and $920 \mathrm{~K}$ for liquid $\mathrm{Co}, \mathrm{Dy}_{0.1} \mathrm{Co}_{0.9}$, and $\mathrm{Dy}_{0.2} \mathrm{Co}_{0.8}$ alloys, respectively. The $\theta$ values obtained for liquid $\mathrm{Er}_{0.1} \mathrm{Co}_{0.9}, \mathrm{Er}_{0.2} \mathrm{Co}_{0.8}, \mathrm{Gd}_{0.1} \mathrm{Co}_{0.9}$, and $\mathrm{Gd}_{0.2} \mathrm{Co}_{0.8}$ alloys are 1340, 790, 1140 , and $730 \mathrm{~K}$, respectively. The rapid decrease in $\chi$ of liquid RE-Co (RE $=\mathrm{Dy}, \mathrm{Er}, \mathrm{Gd})$ alloys on the Co-rich side corresponds to the rapid decrease in $\theta$ which results from the reduction of exchange d-d interaction between Co ions.

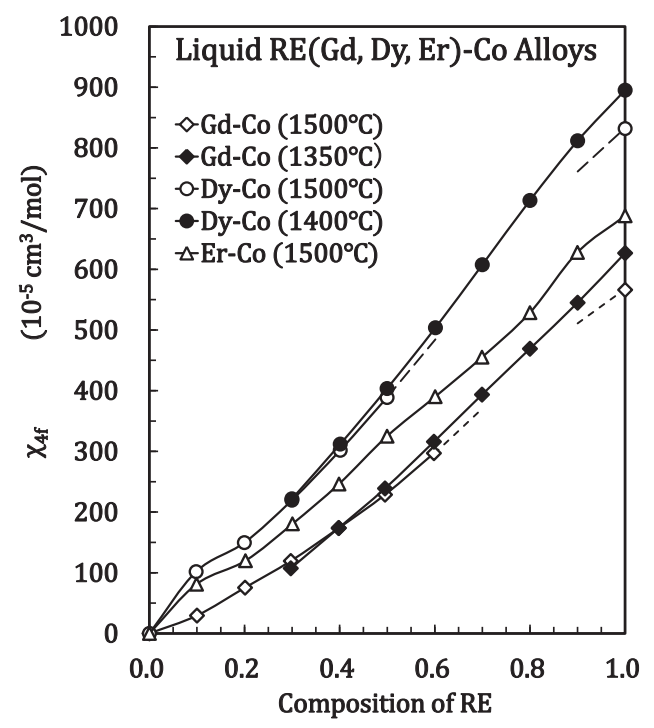

Fig. 4. Dependence of $\chi 4 \mathrm{f}$ on composition for liquid $\mathrm{RE}_{c} \mathrm{Co}_{1-c}(\mathrm{RE}=\mathrm{Dy}, \mathrm{Er}, \mathrm{Gd})$ alloys. The solid lines and dashed lines are drawn as guides for eye.

As shown in Fig. 5, we integrate the trends of $P_{\text {eff }}$ (RE ions) in liquid RE-Co (RE $=$ Dy, Er, Gd, Pr, Ce) alloys. The $P_{\text {eff }}$ values of Ce and Pr ions were estimated from their data $[2$, $3]$ by the same manner. The $P_{\text {eff }}$ values of Dy and Er ions are approximately 10.9 and 9.9, which are somewhat larger than the theoretical prediction of 10.6 and 9.6 estimated for $\mathrm{Dy}^{3+}$ and $\mathrm{Er}^{3+}$ ions [16], respectively. The $P_{\text {eff }}$ value of $\mathrm{Gd}^{3+}$ ions is approximately 8.9, which is larger than the theoretical prediction of 7.9 estimated for $\mathrm{Gd}^{3+}$ ions [16]. A swell in $P_{\text {eff }}$ for $\mathrm{Gd}^{3+}$ ions on the Gd-rich side may be attributable to the assumption of $\theta=0$. If we can obtain an accurate $\theta$ value, we apply the general equation of $P_{\text {eff }}=\left[3 k_{\mathrm{B}}(T-\theta) \chi_{4 \mathrm{f}} / N_{\mathrm{A}}\right]^{1 / 2}$ for the analysis of $P_{\text {eff. }}$.

It is supposed that metallic RE alloys can be represented by an assembly of $\mathrm{RE}^{3+}$ ions embedded in a sea of conduction electrons [17]. In the case of heavy RE-TM alloys [17], the spin of TM ion lying near $\mathrm{Gd}^{3+}$ ion take the orientation contrary to the total spin of $\mathrm{Gd}^{3+}$ ion. Therefore, a gradual decrease in $P_{\text {eff }}$ for liquid RE-Co (RE $=$ Dy, Er, Gd) alloys may be attributed to the alignment of antiparallel moment due to the indirect $\mathrm{d}-\mathrm{f}$ interaction by way of the conduction electrons [17]. It is well known that liquid Ce-Co alloys give rise to the continuous transition of $\mathrm{Ce}^{n^{+}}$ions with increasing Co content $[2,3]$. The trend of $P_{\text {eff }}$ for $\mathrm{Ce}^{n+}$ ions in liquid Ce-Co alloys is closely related to the continuous transition of Ce ions into the nonmagnetic state. We confirmed the transition of $\mathrm{Ce}^{3+}$ to $\mathrm{Ce}^{4+}$ ions by this treatment. On the other hand, the Pr ions are stable in liquid Pr-Co alloys. On the Co-rich side, the $P_{\text {eff }}$ values correspond to the intermediate state between $\mathrm{Co}^{+}$and $\mathrm{Co}^{2+}$ ions with the 
total spin $S=1$ and 3/2, respectively. This indicates that the s-d interaction dominates the magnetic susceptibilities of liquid RE-Co alloys on the Co-rich side.

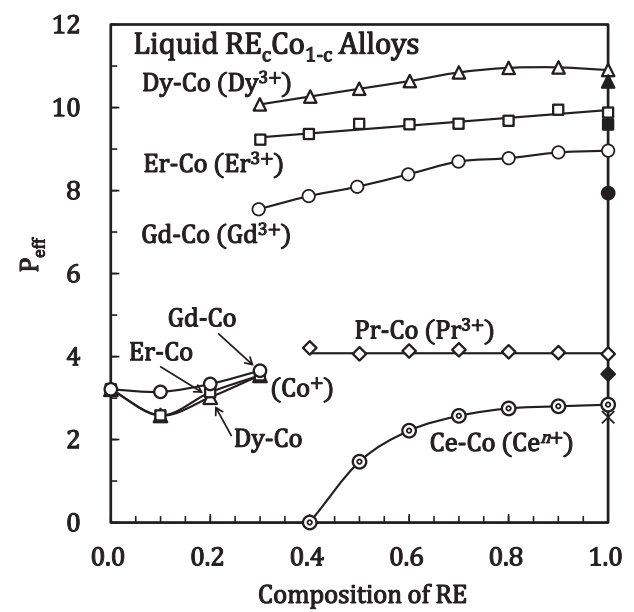

Fig. 5. Dependence of $P_{\text {eff }}$ on composition for RE ( $\left.\mathrm{RE}=\mathrm{Dy}, \mathrm{Er}, \mathrm{Gd}, \mathrm{Pr}, \mathrm{Ce}\right)$ and Co ions obtained in liquid RE-Co alloys. The closed traiangle, closed square and so on plotted on the RE side are the calculated values due to Van Vleck [16]. The solid lines are drawn as guides for the eye.

\section{Conclusions}

Based on the compositional dependence of $\chi$ for liquid La-Co alloys, those for liquid DyCo and Er-Co alloys can be divided into the two main terms due to the $4 \mathrm{f}-$ and $3 \mathrm{~d}$-lectrons. Using the Curie and the Curie-Weiss law, $P_{\text {eff }}$ for Dy, Er, and Co ions can be estimated from the temperature dependence of $\chi$ divided partially. By the addition of $\mathrm{Co}$, the gradual decrease in $P_{\text {eff }}$ of Dy and Er ions in these liquid alloys may be attributed to the indirect d-f interaction by way of the conduction electrons. In the liquid RE-Co systems, the $4 \mathrm{f}$-electron states involved with the s-f interaction predominates steadily over the wide composition rage.

\section{References}

1. G. Busch, H. -J. Güntherodt, H. U. Künzi, H. A. Meier, L. Schlapbach, J. Phys. Colloque 35, C4-329 (1974)

2. L. Schlapbach, Phys. Cond. Matter 18, 189 (1974)

3. V. V. Singer, A. E. Vafin, L. M. Sandratskii, L. P. Mokhracheva, S. V. Konovalov, I. Z. Radovskii, P. V. Geld, Phys. Status. Solidi A 117, 577 (1990)

4. S. Tahara, S. Ohno, and T. Okada: J. Phys. Soc. Jpn. 78, 074702 (2009)

5. S. Ohno, H. Shimakura, S. Tahara, T. Okada, J. Phys. Soc. Jpn. 84, 014706 (2015)

6. M. Müller, E. Huber, H. -J. Güntherodt, J. Phys. Colloque. C5, 260 (1979)

7. C. Kittel, Introduction to Solid State Physics, 5th ed. (Wiley, New York, 1953) Chs. 14 and 15

8. G. A. Bain, J. F. Berry, J. Chem. Educ. 85, 532 (2008)

9. L. B. Mendelsohn, F. Biggs, J. B. Mann, Phys. Rev. A2, 1130 (1970)

10. J. Banhart, H. Ebert, J. Voitländer, H. Winter, J. Magn. Magn. Mater. 61, 221 (1986) 
11. T. E. Faber, An Introduction to the Theory of liquid Metals (Cambridge University Press, London, 1972) Chs. 1 and 4

12. S. D. Silverstain, Phys. Rev. 130, 1703 (1963)

13. D. Pines, Elementary Excitations in Solids (W. A. Benjamin, New York, 1964) Chap. 5

14. P. W. Anderson, Phys. Rev. 124, 41 (1961)

15. N. H. March, C. M. Sayers, Adv. Phys. 28, 1 (1979)

16. J. H. Van Vleck, The Theory of Electric and Magnetic Susceptibilities (Oxford University Press, London, 1932) p. 226

17. K. N. R. Taylor, Adv. Phys. 20, 551 (1971) 